EUROPEAN ORGANIZATION FOR NUCLEAR RESEARCH

European Laboratory for Particle Physics

Large Hadron Collider Project

LHC Project Report 509

\title{
PLASTIC STRAIN INDUCED DAMAGE EVOLUTION AND MARTENSITIC TRANSFORMATION IN DUCTILE MATERIALS AT CRYOGENIC TEMPERATURES
}

\author{
C. Garion and B.T. Skoczen
}

\begin{abstract}
The Fe-Cr-Ni stainless steels are well known for their ductile behaviour at cryogenic temperatures. This implies development and evolution of plastic strain fields in the stainless steel components subjected to thermo-mechanical loads at low temperatures. The evolution of plastic strain fields is usually associated with two phenomena: ductile damage and strain induced martensitic transformation. Ductile damage is described by the kinetic law of damage evolution. Here, the assumption of isotropic distribution of damage (microcracks and microvoids) in the Representative Volume Element (RVE) is made. Formation of the plastic strain induced martensite (irreversible process) leads to the presence of quasi-rigid inclusions of martensite in the austenitic matrix. The amount of martensite platelets in the RVE depends on the intensity of the plastic strain fields and on the temperature. The evolution of the volume fraction of martensite is governed by a kinetic law based on the accumulated plastic strain. Both of these irreversible phenomena, associated with the dissipation of plastic power, are included into the constitutive model of stainless steels at cryogenic temperatures. The model is tested on the thin-walled corrugated shells (known as bellows expansion joints) used in the interconnections of the Large Hadron Collider, the new proton storage ring being constructed at present at CERN.
\end{abstract}

LHC Division

Presented at the 2001 Cryogenic Engineering Conference and International Cryogenic Materials Conference CEC/ICMC 2001

16-20 July 2001, Madison, Wisconsin, USA

\footnotetext{
Administrative Secretariat

LHC Division

CERN

CH - 1211 Geneva 23

Switzerland

Geneva, 19 October 2001
} 


\title{
PLASTIC STRAIN INDUCED DAMAGE EVOLUTION AND MARTENSITIC TRANSFORMATION IN DUCTILE MATERIALS AT CRYOGENIC TEMPERATURES
}

\author{
C. Garion, B.T. Skoczen \\ CERN, LHC Division \\ CH-1211, Geneva 23, Switzerland
}

\begin{abstract}
The Fe-Cr-Ni stainless steels are well known for their ductile behaviour at cryogenic temperatures. This implies development and evolution of plastic strain fields in the stainless steel components subjected to thermo-mechanical loads at low temperatures. The evolution of plastic strain fields is usually associated with two phenomena: ductile damage and strain induced martensitic transformation. Ductile damage is described by the kinetic law of damage evolution (cf. [1]). Here, the assumption of isotropic distribution of damage (microcracks and microvoids) in the Representative Volume Element (RVE) is made. Formation of the plastic strain induced martensite (irreversible process) leads to the presence of quasi-rigid inclusions of martensite in the austenitic matrix. The amount of martensite platelets in the RVE depends on the intensity of the plastic strain fields and on the temperature. The evolution of the volume fraction of martensite is governed by a kinetic law based on the accumulated plastic strain (cf. [2]). Both of these irreversible phenomena, associated with the dissipation of plastic power, are included into the constitutive model of stainless steels at cryogenic temperatures. The model is tested on the thin-walled corrugated shells (known as bellows expansion joints) used in the interconnections of the Large Hadron Collider, the new proton storage ring being constructed at present at CERN.
\end{abstract}

\section{INTRODUCTION}

The Fe-Cr-Ni stainless steels are commonly used to manufacture components of superconducting magnets and cryogenic transfer lines since they retain their ductility at low temperatures and are paramagnetic. The nitrogen-strengthened stainless steels of series 300 belong to the group of metastable austenitic alloys. Under certain conditions the steels undergo martensitic transformation at cryogenic temperatures that leads to a considerable evolution of material properties and to a ferromagnetic behaviour. The martensitic 
transformations are induced mainly by the plastic strain fields and amplified by the high magnetic fields. The stainless steels of series 300 show at room temperature a classical $\gamma-$ phase of face centred cubic austenite (FCC). This phase may transform either to $\alpha$ ' phase of body centred tetragonal ferrite (BCT) or to a hexagonal $\varepsilon$ phase. The most often occurring $\gamma-\alpha^{\prime}$ transformation leads to formation of the martensite dispersed in the surrounding austenite matrix. In the course of the strain-induced transformation the martensite platelets modify the FCC lattice leading to local distortions. The volume fraction of the martensite $(\xi)$ depends on the chemical composition, temperature, stress state, plastic strains and exposure to a magnetic field. It is well known that solutes like $\mathrm{Ni}$, $\mathrm{Mn}$ and $\mathrm{N}$ considerably stabilise the $\gamma$-phase. For instance the strain-induced martensitic content in the grades $304 \mathrm{LN}, 316 \mathrm{LN}$ at low temperatures is much lower than in the grades 304L, 316L for the same level of plastic strain [3]. Simultaneously, a plastic strain driven by evolution of ductile damage occurs in the stainless steel under thermo-mechanical loads at cryogenic temperatures. This dissipative and irreversible process leads to creation of microcracks and microvoids and to a "softening" of the material. The intensity of isotropic damage fields is described by a scalar parameter $D$, related directly to the accumulated plastic strain $p$. Damage evolution starts above the so-called damage threshold $p_{D}$ corresponding to the minimum accumulated plastic strain necessary to initiate the process. When the damage parameter reaches its critical value $D_{c r}$ an onset and propagation of a macrocrack is observed. Further development of the macrocrack is governed by the fracture mechanics.

Both the martensite inclusions, microcracks and microvoids coexist in the same volume of material (RVE, FIG 1). The volume fraction of martensite is expressed by the following ratio:

$$
\xi=\frac{d V_{\xi}}{d V} ; 0 \leq \xi \leq 1
$$

where $V_{\xi}$ denotes the volume of the martensitic phase and $V$ stands for the total volume.

Damage is represented by a scalar damage variable, that is defined as a surface density of microvoids and microcracks. If $d S_{D}$ denotes the surface of intersection of the micro-voids and micro-cracks with a given plane within the RVE and $d S$ stands for the total intersection surface then the damage parameter is defined as:

$$
D=\frac{d S_{D}}{d S} ; 0 \leq D \leq 1
$$

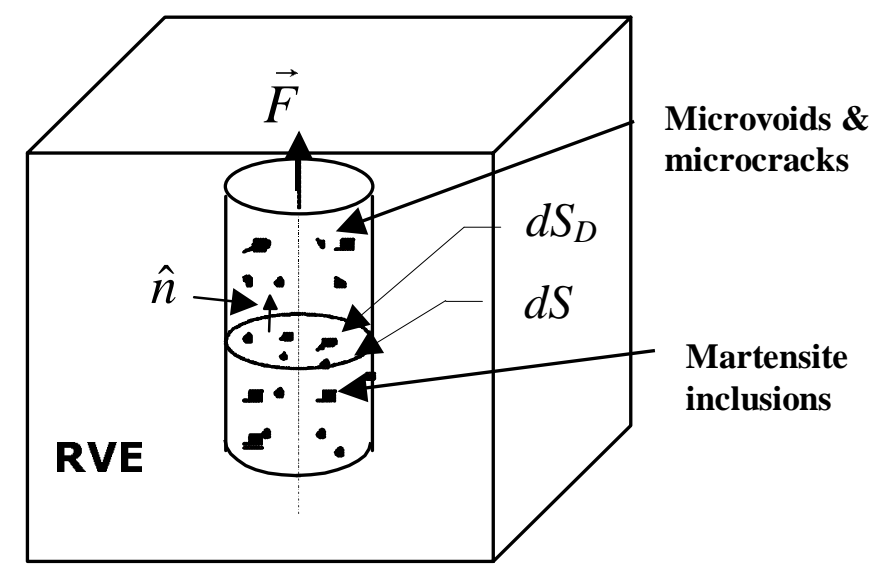

FIGURE 1. The Representative Volume Element with martensite inclusions and damage 
The increase in martensite fraction promoted by plastic deformation can be detected by measuring the magnetic permeability $\mu$. The evolution of $\mu$ at low temperature corresponding to monotonic straining as well as to the cyclic loads for 304L and 316L stainless steels was investigated by Suzuki et al. [4]. Tensile properties of stainless steels at low temperatures are strongly influenced by the plastic strain induced martensitic transformation. As a result of the transformation the initially homogenous $\gamma$-phase loses its homogeneity because of the inclusions of the harder martensite phase. The martensite platelets embedded in the soft austenite matrix provoke local stress concentration and block the movement of dislocations. Therefore the onset of the martensitic transformation leads to an increase of the strain hardening. On the other hand, the intensity of ductile damage can be detected by measurements of the evolution of modulus of elasticity. It is assumed that the elastic properties of the dual-phase material are not modified by the martensitic transformation (the elastic properties of the martensite and of the austenite are quite similar, cf. [5], [6]). Since the effective modulus of elasticity is defined as (cf. [1]):

$$
\tilde{E}=E(1-D)
$$

the evolution of $D$ can be identified via measurements of $\tilde{E}$ on the unloading paths. A simple monotonic tensile test is sufficient to determine the evolution of damage associated with the accumulated plastic strain. Thus, both the intensity of martensitic transformation and the evolution of damage fields can be measured by using the simple and well established methods.

In the present paper the relevant kinetic evolution laws will be formulated and the complete set of constitutive equations allowing analysis of both phenomena at cryogenic temperatures will be developed (cf. [2]). The constitutive model is tested on the thinwalled corrugated shells (bellows expansion joints), used in the interconnections of the LHC at CERN, subjected to thermo-mechanical loads at cryogenic temperatures.

\section{KINETICS OF MARTENSITIC TRANSFORMATION}

Transformation kinetics has been developed by Olson and Cohen [7]. The authors attribute the strain-induced martensite nucleation sites to the shear-band intersections (the shear-bands being in the form of $\varepsilon^{\prime}$ martensite, mechanical twins or stacking-fault bundles). The analysis leads to the following equation for the volume fraction of martensite versus plastic strain:

$$
\xi_{\alpha^{\prime}}=1-\exp \left\{-\beta[1-\exp (-\alpha \varepsilon \mathrm{p})]^{\mathrm{n}}\right\}
$$

where $\alpha$ represents the rate of shear-band formation, $\beta$ represents the probability that a shear-band intersection will become a martensite site and $n$ is a fixed exponent. The transformation curves (volume fraction of martensite versus plastic strain) show a typical sigmoidal shape with saturation levels below $100 \%$ (FIG. 2). Olson and Cohen developed a one-dimensional model for the kinetics of martensitic transformation, called OC model. The evolution of the volume fraction of martensite as a function of plastic strain is derived by considering the shear band formation, the probability of shear-band intersections and the probability of an intersection generating a martensitic embryo. In this model, only temperature and plastic strain control the martensite evolution. Different improvements have been brought to this model, covering the influence of the stress state [5] and the strain 
rate [6]. However, a considerable number of parameters have to be identified for these models. In the present paper, a simplified model - developed by Garion and Skoczen [2] for cryogenic applications - is applied. The volume fraction of martensite $\xi$ can be presented in the following form:

$$
\xi=\xi(\mathrm{p}, \mathrm{T}, \stackrel{\dot{\varepsilon}}{\mathrm{p}}, \sigma)
$$

where $\mathrm{p}$ is the accumulated plastic strain defined by:

$$
\mathrm{p}=\int_{0}^{\mathrm{t}} \sqrt{\frac{2}{3} \dot{\varepsilon}^{\mathrm{p}}: \dot{\varepsilon}^{\mathrm{p}}} \mathrm{d} \tau
$$

$\underset{\dot{\varepsilon}}{\mathrm{p}}$ denotes the plastic strain rate and $\underset{\sigma}{=}$ is the stress tensor. Under isothermal conditions and for a given strain rate, the classical sigmoidal curve has the following form (FIG. 2):

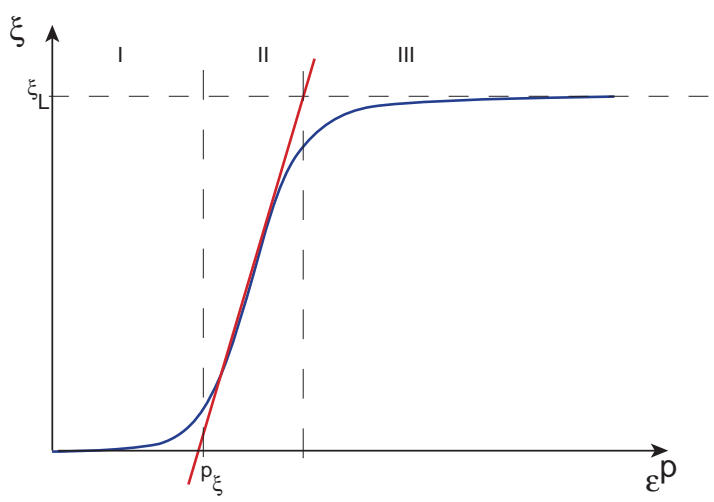

FIGURE 2. Volume fraction of martensite versus plastic strain at cryogenic temperatures $(4 \mathrm{~K}, 77 \mathrm{~K})$

The curve is decomposed into 3 phases: phase $I$ corresponds to a nonlinear increase of the martensitic content with the plastic strain, phase $I I$ - the $\alpha^{\prime}$ volume fraction $(\xi)$ is linearly related to plastic deformation $\left(\varepsilon^{\mathrm{p}}\right)$ and phase III corresponds to a saturation effect $\left(\xi_{L}\right)$. A simplified law of evolution of the martensite content has been proposed (cf. [2]) for the phase II under the following form:

$$
\dot{\xi}=\mathrm{A}(\mathrm{T}, \underset{\underline{\sigma}}{\underline{\underline{\varepsilon}}} \stackrel{\mathrm{p}}{\mathrm{p}}) \dot{\mathrm{p} H}\left((\mathrm{p}-\mathrm{p} \xi)\left(\xi_{\mathrm{L}}-\xi\right)\right)
$$

where $\mathrm{A}$ is a function of the temperature, the stress and the plastic strain rate, $\mathrm{p}_{\xi}$ is the accumulated plastic strain threshold (to trigger the formation of martensite), $\xi_{\mathrm{L}}$ is the martensite content limit, over which the martensitic transformation rate is considered equal to 0 . Finally, $H$ represents the Heavyside function $(H(x)=1$ if $x \geq 0 ; H(x)=0$ if $x<0)$.

\section{KINETICS OF EVOLUTION OF DUCTILE DAMAGE}

The damage variable, as introduced by Kachanov [8], has been defined on the basis of the irreversible thermodynamic processes leading to nucleation and growth of the microvoids and micro-cracks in the entire volume of a sample. Here all types of voids and cracks 
(inter- and trans-granular) that spoil integrity of the material are accounted for. Thus, the process of damage development leads by definition to an increase of entropy.

The damage parameter, as defined by Eq. 2, is a non-negative state variable satisfying the following inequality:

$$
0 \leq D \leq D_{c r},
$$

where $D=0$ for a non-damaged material and $D=D_{c r}$ for a total decohesion of the sample (usually $D_{c r} \leq 1$, FIG. 3).

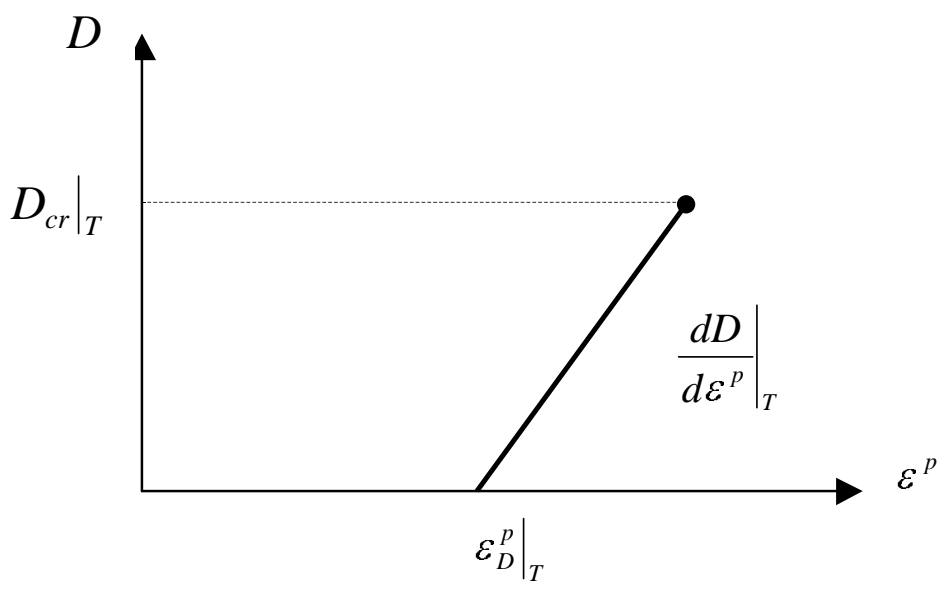

FIGURE 3. Linearized evolution of damage at a given temperature T: $\left.\varepsilon_{D}^{p}\right|_{T}$ denotes damage threshold

Since the damage evolution is a "dissipative" phenomenon similar to the evolution of the plastic strain fields, a similar formulation for the potential of dissipation is applied (a continuous, convex scalar function of the dual variables):

$$
\dot{D}=\frac{\partial F}{\partial Y} \dot{\lambda} ; F=\frac{S}{(s+1)(1-D)}\left(\frac{Y}{S}\right)^{s+1}
$$

where $S$ represents the strength energy of damage (material parameter) and $s$ has to be identified by using the experimental data. $Y$ denotes the strain energy density release rate expressed by:

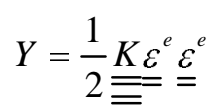

Such a formulation yields the following kinetic law of damage evolution (cf. Lemaitre [1]):

$$
\dot{D}=\left(\frac{Y}{S}\right)^{s} \dot{p} H\left(p-p_{D}\right)
$$

where $p_{D}$ is the so-called damage threshold.

\section{CONSTITUTIVE EQUATIONS OF ELASTO-PLASTIC MATERIAL WITH EVOLUTION OF DAMAGE AND MARTENSITE CONTENT}

The variables associated with the state functions can be derived from a state potential. It is assumed that the state potential is assimilated with the Helmholtz free 
energy and that for an elasto-plastic material and an isothermal process it can be expressed as:

$$
\Psi=\Psi(\underline{\underline{\varepsilon}}, r, \underline{\underline{\alpha}}, D)=\Psi\left(\underline{\underline{\varepsilon}}-\underline{\underline{\varepsilon^{p}}}-\underline{\underline{\varepsilon}}^{t h}-\underline{\underline{\varepsilon}}^{P T}, r, \underline{\underline{\alpha}}, D\right)
$$

or, in the developed form:

$$
\Psi=\frac{1}{\rho}\left(\frac{1}{2}(1-D) \underline{\underline{\underline{\underline{K}}}} \underline{\underline{\underline{\varepsilon}}} \underline{\underline{\underline{\varepsilon}}} \underline{\underline{\varepsilon}}+\frac{1}{3} H(\xi) \underline{\underline{\alpha \alpha}}\right)+\Psi_{r}
$$

where $\underline{\varepsilon}^{P T}$ is the distortional strain field associated with the phase transformation, $H$ is the kinematic hardening modulus, $\underline{\alpha}$ is the back strain and $\Psi_{r}$ stands for the part of the Helmholtz potential associated with the isotropic hardening. Here, the assumption is made that both the density $\rho$ and the elastic stiffness tensor $\underline{\underline{\equiv}}$ depend weakly on the volume fraction of martensite $\xi$. Based on the Helmholtz state potential the following set of constitutive equations can be derived:

- kinetics of the martensitic transformation:

$$
\dot{\xi}=A\left(T, \underline{\underline{\sigma}} \stackrel{\dot{\varepsilon}^{p}}{=} \dot{p} H\left(\left(p-p_{\xi}\right)\left(\xi_{L}-\xi\right)\right)\right.
$$

- kinetics of damage evolution:

$$
\dot{D}=\left(\frac{Y}{S}\right)^{s} \dot{p} H\left(p-p_{D}\right)
$$

- the constitutive law:

$$
\underline{\underline{\sigma}}=\underline{\underline{\underline{K}}}:\left(\underline{\underline{\varepsilon}}-\underline{\underline{\varepsilon}}^{p}-\underline{\underline{\varepsilon}}^{\text {th }}-\underline{\underline{\varepsilon}}^{P T}\right)
$$

- the yield surface:

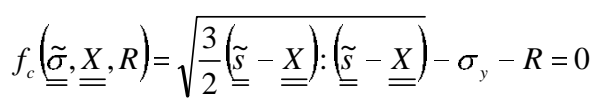

- the normality rule:

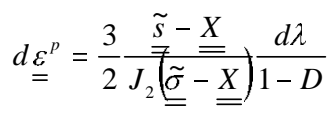

- the kinematic hardening:

$$
\underline{\underline{X}}=\frac{2}{3} H(\xi) \stackrel{\dot{\varepsilon}^{p}}{=}(1-D)=\frac{2}{3} H(\xi) \stackrel{\dot{\alpha}}{=}
$$

where:

$$
H(\xi)=C(\xi)+3 \beta(1-\xi)\left[\mu_{M T}(\xi)-\mu_{t 0}\right]
$$

- the isotropic hardening:

$$
\dot{R}=b(\xi)\left[R_{\infty}(\xi)-R\right] \dot{p}(1-D)=b(\xi)\left[R_{\infty}(\xi)-R\right] \dot{r}
$$

where:

$$
\begin{aligned}
& b(\xi)=(1-\xi)(1-\beta) \\
& R_{\infty}(\xi)=3\left[\mu_{M T}(\xi)-\mu_{t 0}\right]
\end{aligned}
$$

Here, $C(\xi)$ is the hardening modulus of biphase material, $\beta$ stands for the Bauschinger parameter and $\mu_{M T}$ denotes the tangent modulus resulting from the Mori-Tanaka homogenisation. Also, in the above given set of equations the concept of effective stress and effective deviatoric stress has been used:

$$
\underline{\underline{\tilde{\sigma}}}=\frac{\underline{\underline{\sigma}}}{1-D} \quad ; \stackrel{\tilde{s}}{=} \frac{\stackrel{s}{=}}{1-D}
$$


It is worth pointing out that no coupling between the evolution of damage and volume fraction of martensite is assumed at the level of the kinetic evolution laws. This assumption may turn out to be too strong and therefore needs to be verified via further experimental research under cryogenic conditions.

\section{NUMERICAL IMPLEMENTATION AND RESULTS}

The model presented in the previous chapter has been numerically implemented in the FE code CASTEM 2000. The method of the type "return mapping" is used to integrate the constitutive equations for an active plastic process. The return mapping algorithm is based on the elastic-plastic split, by first integrating the elastic equations to obtain an elastic predictor, which is used as initial condition for the plastic return. The constitutive model is tested on the thin-walled stainless steel corrugated shells, known as bellows expansion joints, used in the interconnections of the LHC.

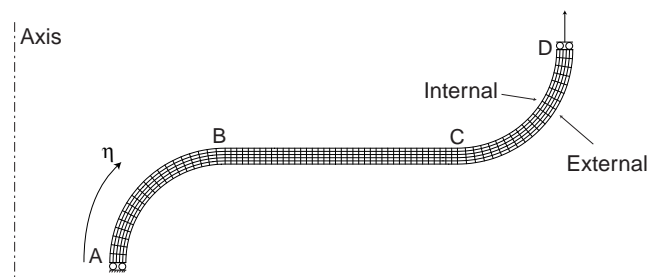

FIGURE 4. Model of half-convolution of a cryogenic bellows

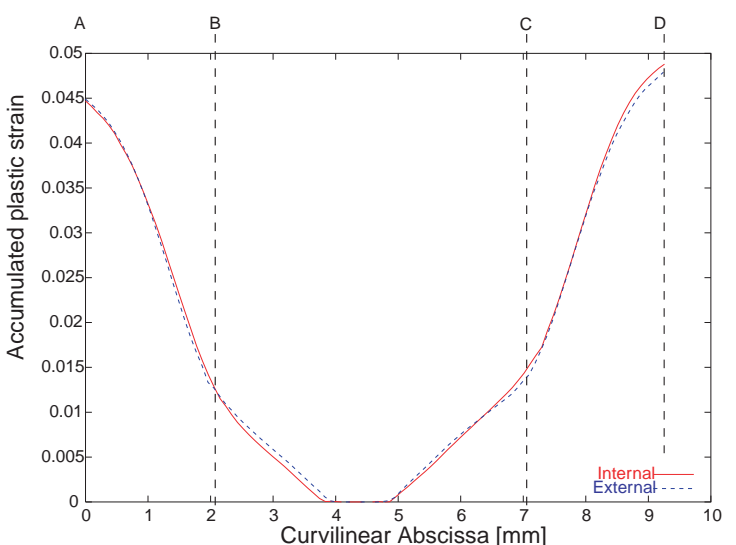

FIGURE 5. Accumulated plastic strain (p) along the convolution

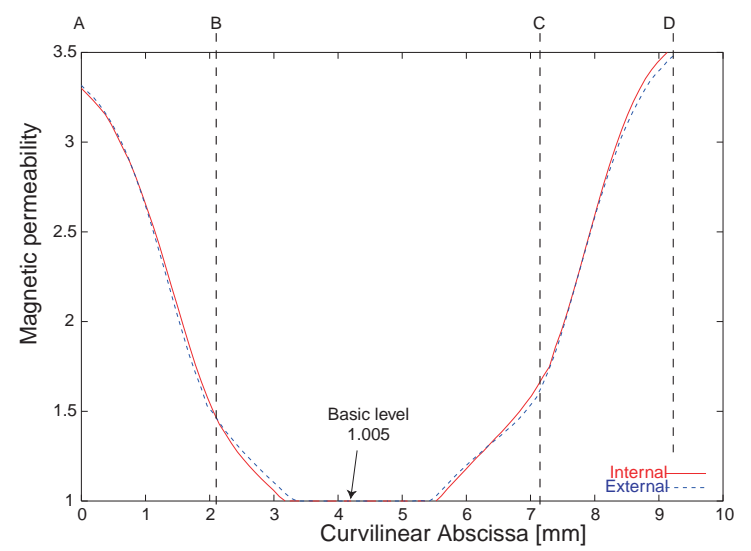

FIGURE 7. Magnetic permeability $(\mu)$ along the convolution

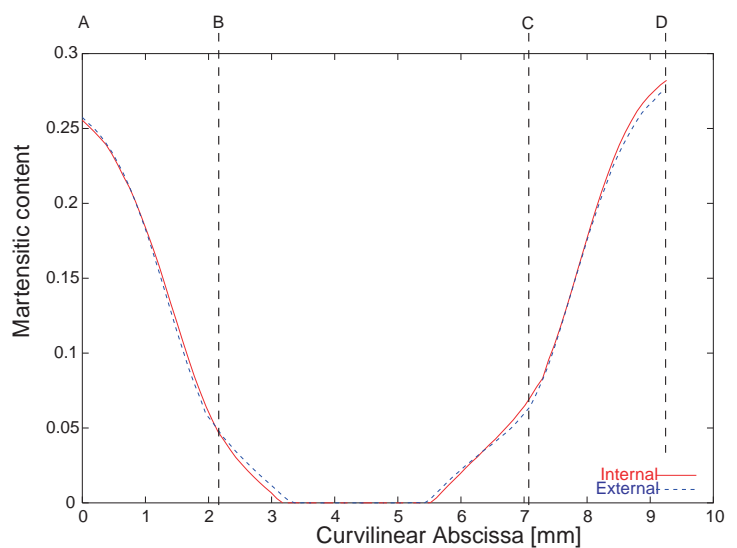

FIGURE 6. Martensite content $(\xi)$ along the convolution

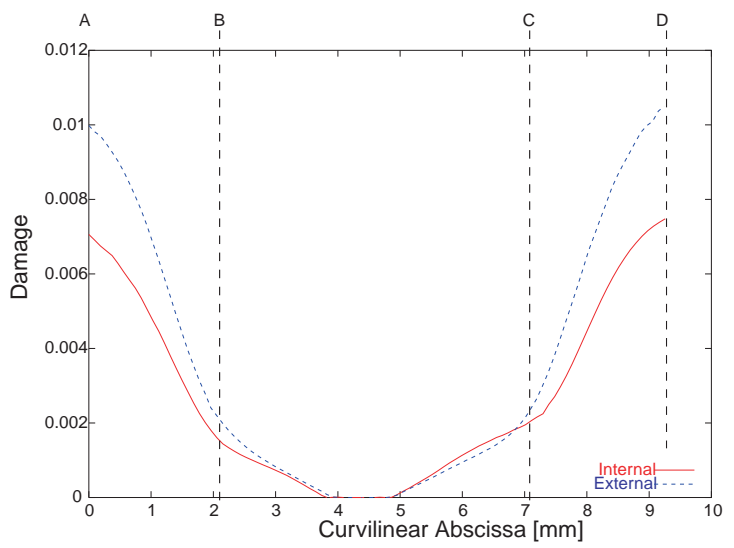

FIGURE 8. Damage (D) along the the convolution ( $\mathrm{p}_{\mathrm{D}}$ is assumed equal to 0 ) 
The bellows are subjected to particularly severe loads (thermomechanical cycles between ambient and $1.9 \mathrm{~K}$, internal pressure up to $2 \mathrm{MPa}$ ) therefore the analysis of accumulation of plastic strains, damage and volume fraction of martensite is of particular interest for the reliability of the collider. Half convolution of a typical bellows is shown in FIG. 4 (axisymmetric FE model). The bellows is subjected to an axial stretch of $185 \%$ of its initial length at $77 \mathrm{~K}$. The accumulation of plastic strains and the corresponding evolution of the volume fraction of martensite is shown in FIG.5 and FIG.6, respectively. The magnetic permeability (corresponding to the field of $1000 \mathrm{Oe}$ ) is presented in FIG.7. Finally, the evolution of the damage parameter along the convolution is shown in FIG.8.

\section{CONCLUSIONS}

In the present paper a constitutive model of plastic flow, accompanied by the strain induced martensitic trasformation and the evolution of isotropic ductile damage at cryogenic temperatures, has been presented. The description of the plastic strain induced martensitic transformation is based on the kinetics developed by Olson and Cohen. However, a new kinetic law of evolution of the volume fraction of martensite, corresponding to phase II of the sigmoidal transformation curve, has been proposed. The evolution of isotropic ductile damage is based on the kinetic law developed by Lemaitre. The accumulation of ductile damage is also driven by the evolution of plastic strains. Both the martensitic transformation and the evolution of ductile damage are irreversible and dissipative phenomena that occur at cryogenic temperatures and lead to the coexistence of martensite inclusions, microcracks and microvoids. The mutual influence of phase transformation and ductile damage is not yet well determined. Therefore, both kinetic laws are not coupled. This particular problem needs further extensive study.

The application of the numerical model to the analysis of evolution of plastic strains, volume fraction of martensite and ductile damage in the cryogenic bellows, designed for the interconnections of the LHC, shows a strong concentration of damage and martensite at root and at crest of the convolutions. This yields a risk of propagation of a macrocrack once the damage parameter will reach its critical level $\left(D_{c r}\right)$. Also, an enhanced level of the magnetic permeability at root and at crest can be expected. Both effects have to be taken into account when designing a reliable cryogenic mechanical compensation system.

\section{REFERENCES}

1. Lemaitre, J., A Course on Damage Mechanics, Springer Verlag, Berlin, 1992.

2. Garion, C., Skoczen, B., ASME Journ. Appl. Mech., under review, 2001.

3. Morris, J. W., Chan, J. W. and Mei, Z., Cryogenics 32 ICMC supplement, pp. $78-85$ (1992).

4. Suzuki, K., Fukakura, J. and Kashiwaya, H., Journ. of Test. and Eval. 16, pp. 190-197 (1988).

5. Stringfellow, R. G., Parks, D. M. and Olson, G. B., Acta. Metall. Mater. 40, pp. 1703-1716 (1992).

6. Levitas, V.I., Idesman, A.V. and Olson, G.B., Acta. Mater. 47, pp. 219-233 (1999).

7. Olson, G. B. and Cohen, M., Metallurgical Transactions 6A, pp. 791-795 (1975).

8. Kachanov, L.M., Introduction to Continuum Damage Mechanics, Martinus Nijhoff Dordrecht, 1986. 